\title{
Internet-basierte Umwelt- bildung: Eine experimentelle Studie zur Anwendung des Online-Simulationsspiels SimUlme im Schulunterricht
}

\section{Ralf Hansmann, Stefan Hesske, Olaf Tietje und Roland Scholz}

Ein internetbasierendes Simulationsspiel zu ökologisch-ökonomischen Konsequenzen des Konsumverhaltens im Ernährungsbereich wurde entwickelt, um ökologische Lerninhalte spielerisch und anschaulich zu vermitteln. In einem Experiment erhielten 12 Schulklassen Unterricht zu Umweltfolgen des Nahrungsmittelkonsums, 6 Klassen mit Anwendung des SimUlme Spiels (experimentelle Bedingung), 6 Klassen ohne (Vergleichsbedingung). Verständlichkeit, aktive Selbstbeteiligung und Interessantheit des Unterrichts wurden von den Schülerinnen und Schülern in der experimentellen Bedingung signifikant höher eingeschätzt als in der Vergleichsbedingung. Der Tendenz nach erreichte der Unterricht mit SimUlme zudem stärkere positive Veränderungen von Einstellungen im Ernährungsbereich. Das Wissen wurde in beiden Bedingungen verbessert, aber in unterschiedlichem Ausmass hinsichtlich verschiedener, sich ergänzender Aspekte. Die Ergebnisse sprechen für die Anwendung geeigneter Internet-Spiele im Schulunterricht.

\section{Einleitung}

Der Einsatz von Computern im Schulunterricht fördert den Erwerb von Computerkenntnissen, was für die späteren beruflichen Chancen und die berufliche Praxis der Schülerinnen und Schüler bedeutsam ist. Auch der kompetente Umgang mit dem Internet als einer mittlerweile elementaren Kulturtechnik sollte hierbei vermittelt werden (Kerres, 2000a). Durch den Einsatz von Computern kann man zudem andere, vom Computer an sich unabhängige Lerninhalte vermitteln. Forschungsergebnisse in Bezug auf den Erfolg computerunterstützter Instruktion im Vergleich zu traditionellen Lernformen sind nicht einheitlich, aber sprechen im Mittel für eine grössere Effektivität von computerunterstütztem Lernen (Glowalla \& Häfele, 1995). Die Schwankungen zwischen den Ergebnissen der Einzelstudien zu unterschiedlichen computerbasierten Lernmedien zeigen, dass die Gestaltung von computerunterstützten Lernumgebungen 
für deren Erfolg entscheidend ist (Kerres, 2000a, 2000b). Der Einsatz von Computern besitzt somit bei angemessener Gestaltung und geeigneter Einbettung in ein umfassendes Instruktionsdesign (Reigeluth, 1983) durchaus ein Verbesserungspotential für die Aus- und Weiterbildung (Kerres \& Jechle, 2000).

Die gesellschaftliche Bedeutung des Umweltschutzes spiegelt sich an den Schweizer Schulen in der Durchführung einer Vielzahl von umweltbezogenen Schulprojekten wider (Hesske \& Frischknecht-Tobler, 1999, 2000; Kyburz-Graber, Rigendinger, Hirsch Hadorn \& Werner Zentner, 1997). Ziel von Umweltunterricht an Schulen ist, nachhaltiges, umweltgerechtes Verhalten und entsprechende positive Einstellungen und Werte an die Schülerinnen und Schüler zu vermitteln (Kyburz-Graber \& Schlüter, 2001). Dies trägt der Bedeutung des Umweltschutzes für unsere Gesellschaft Rechnung (Kaufmann-Hayoz \& Di Giulio, 1996). Umweltgerechtes Verhalten soll hierbei sowohl in der Gegenwart und in privaten Lebensbereichen der Schülerinnen und Schüler gefördert werden, als auch im Hinblick auf deren späteres berufliches Verhalten. Umweltunterricht ist somit eine zukunftsorientierte Stütze für eine nachhaltige Entwicklung unserer Gesellschaft.

Eine Kombination von Umweltunterricht mit aktivem Einsatz von Computern erscheint sinnvoll, da so ein bestehendes Interesse von Schülern für den einen oder den anderen Bereich synergetisch den Erwerb von Kenntnissen im jeweils anderen Bereich fördern kann. Zur Bezeichnung von Lernprogrammen, mit deren Hilfe Lerninhalte an die Lernenden spielerisch vermittelt werden können, wurde in jüngster Zeit der Begriff bzw. die Wortneuschöpfung Edutainment (Bauer, 1995) eingeführt. Dieser Begriff beinhaltet autonome Aktivität, Kreativität und intrinsisches Interesse der oder des Lernenden am inhaltlichen und gleichzeitig praktischen Umgang mit einem Thema. Die mit dem Begriff Spielen verbundene positive emotionale Komponente kann als eine intrinsische Motivation betrachtet werden, die mit einem förderlichen und angenehmen Aktivierungsgrad einhergeht und eine ganzheitliche und konzentrierte Teilnahme am Lernprozess ermöglicht. So wird z.B. in der Verhaltensbiologie Spielen als ein natürliches Verhaltensprogramm zum Erwerb von Wissen und Fähigkeiten bezeichnet. Die Motivation zur Ausführung des Spielverhaltens besteht hierbei in einer intrinsischen Befriedigung des Spielenden, was notwendig ist, da eine externe Gratifikation für die hiermit verbundenen, vielschichtigen Lernerfahrungen zumeist erst in fernerer Zukunft erfolgt (Hassenstein, 2001). ComputerLernspiele sind vor diesem Hintergrund möglicherweise geeignet, Lernmotivationen von Schülerinnen und Schülern zu fördern.

Im vorliegenden Experiment wird Schulunterricht zu umweltgerechtem Ernährungsverhalten mit Anwendung des Online Computersimulationsspiels SimUlme (Simulation der Umweltfolgen von Lebensmitteleinkäufen) verglichen mit Unterricht zum gleichen Thema, aber ohne Anwendung dieses Lernspiels. SimUlme kann hierbei allgemein als Repräsentant für eine computerunterstützte Lernumgebung betrachtet werden, die bestimmte, vor dem Hintergrund beste- 
hender Theorien und Forschungsergebnisse sinnvoll erscheinende Merkmale besitzt. Ziel des Unterrichts war in beiden Bedingungen die Vermittlung von handlungsrelevantem Umweltwissen im Bereich Lebensmittelkonsum und von positiven umweltbezogenen Einstellungen, also letztlich die Förderung von umweltgerechtem Verhalten.

\section{SimUlme - Ein Spiel zu Umweltfolgen von Lebensmitteleinkäufen}

SimUlme ist ein Simulationsspiel, das auf der Grundlage von Kaufentscheidungen bei Lebensmitteleinkäufen Szenarien der wirtschaftlichen und ökologischen Entwicklung in der Schweiz zeigt. Die Auswirkungen des Kaufverhaltens werden in SimUlme verstärkt dargestellt, aber in ihrer Richtung realistisch. Das Lernspiel ist unter der Adresse http:/andros.ethz.ch/ks/simulme.asp im Internet frei zugänglich und jederzeit online spielbar. Dort ist auch eine ausführliche Erklärung des Spiels mit Hintergrundinformationen zu finden. Eine Grundlage für das Spiel ist u.a. die modulare Ökobilanz von Jungbluth (2000) über die Umweltbelastungen durch Produktion, Verarbeitung, Transport, Verpackung und Konsum von Gemüse und Fleisch. Auf ihrer Grundlage wurde auch die Website ULME erstellt (http://www.ulme.uns.umnw.ethz.ch), die eine Berechnung der Umweltfolgen von Lebensmitteleinkäufen ermöglicht und eine informative Ergänzung zu SimUlme darstellt.

Die oder der Spielende geht im SimUlme Spiel sechsmal einkaufen. Zuerst im Jahr 2000, dann fünf Jahre später im Jahr 2005, wieder fünf Jahre später, usw. ... bis zum Jahr 2025. Die Einkäufe werden als durchschnittliches Einkaufsverhalten für die Dauer von fünf Jahren interpretiert. Nach jedem Einkauf wird eine ökologisch-ökonomische Simulation gezeigt, welche sich als Folge des Einkaufsverhaltens ergibt. Hierzu wird die Entwicklung von je vier wirtschaftlichen und ökologischen Grössen dargestellt. Die vier ökonomischen Grössen sind: 1) Arbeitslosenquote, 2) Anzahl der Beschäftigten in der Landwirtschaft, 3) durchschnittliches Einkommen landwirtschaftlicher Betriebe und 4) die Kaufkraftentwicklung, welche auch einen Einfluss auf die Entwicklung des persönlichen Spieleinkommens hat. Die ökologische Entwicklung wird simuliert durch: 5) die Grösse der landwirtschaftlich genutzten Fläche in der Schweiz, 6) die Bodenbelastung der landwirtschaftlich genutzten Fläche, 7) die Verwirklichung artgerechter Tierhaltung in der Landwirtschaft und 8) die globale Umweltbelastung. Die Simulation der Entwicklung beinhaltet für jede Grösse Balkendiagramm, kurzen Begleittext und Bild. Ausgangssituation vor dem ersten Einkauf ist ein entsprechender Überblick über die ökologisch-ökonomische Situation der Schweiz im Jahr 2000.

Ein Einkauf besteht aus der Beantwortung von Fragen zu fünf Bereichen mit ökologisch-ökonomischer Relevanz, wobei die Antworten jeweils durch die 
Zuordnung von Anteilen zu vorgegebenen Möglichkeiten erfolgen. Die fünf Bereiche sind: 1) das Mengenverhältnis des Konsums von Gemüse versus Fleisch, 2) die Herkunft des konsumierten Gemüses, 3) die Anbauart des Gemüses, 4) die Produktionsart des Fleisches und 5) die Herkunft des Fleisches.

\section{Lernpsychologisch relevante Aspekte des Lernspiels SimUlme}

Bei der Entwicklung und Gestaltung des umweltpädagogischen Spiels SimUlme wurden Erkenntnisse der sozial-kognitiven Lerntheorie (Bandura, 1979), der Lehr/Lernforschung, umweltpsychologische Überlegungen sowie Theorien und Ergebnisse der psychologischen Forschung zum Erwerb und zur Verhaltenswirksamkeit von Einstellungen berücksichtigt. In diesem Zusammenhang sind die folgenden Aspekte der Gestaltung von SimUlme hervorzuheben: 1) die Übernahme der Rolle eines Trendsetters, 2) die Rolle als Managerin bzw. Manager mit Verantwortung für das Gesamtsystem, 3) die Kombination von Lernen durch Feedback mit instruierenden Erklärungen, 4) die Verstärkung emotionaler Aspekte durch fotografische Abbildungen und 5) die multiple, interaktive Repräsentation von Prozessen und Systemzuständen.

\section{ad 1. Die übernahme der Rolle eines Trendsetters}

Umweltschädigendes Verhalten wird dadurch begünstigt, dass die Folgen eigener Handlungen häufig nicht erkannt werden (Ernst, 1997). Um die Bedeutsamkeit des Einkaufsverhaltens der oder des Spielenden für Umwelt und Ökonomie zu verstärken, wurde ihr oder ihm die Rolle eines Trendsetters für das Einkaufsverhalten anderer zugewiesen. Zu Beginn des Spiels wird die oder der Spielende aufgefordert zu definieren, in welchem Ausmass mit dem eigenen Einkaufsverhalten ein gleichgerichteter Einfluss auf das Einkaufsverhalten aller Schweizer Konsumentinnen und Konsumenten ausgeübt wird bzw. welcher prozentuale Anteil der Bevölkerung das eigene Einkaufsverhalten nachahmen soll. Im Spiel wird so vermittelt, dass man ein soziales Modell (Bandura, 1979), ein Vorbild, für andere Verbraucher ist. Entsprechende indirekte Auswirkungen des eigenen Verhaltens auf die Umwelt werden möglicherweise häufig nicht oder nur unzureichend erkannt. Dies, obwohl gesellschaftliche Innovation und sozialer bzw. ökologischer Wandel gerade auch durch soziale Einflussprozesse erreichbar sind (Hansmann, 2001; Moscovicci \& Faucheux, 1972; Mugny, 1982).

\section{ad 2. Die Rolle als Managerin bzw. Manager mit Verant- wortung für das Gesamtsystem}

In SimUlme beeinflusst man durch sein Spielverhalten die ökologische und ökonomische Entwicklung der Schweiz. Die Kontrollmacht über die entsprechenden Systemgrössen kann zur Identifikation mit der Rolle des systemverantwort- 
lichen Managers führen. Dies sollte mit verstärkter Betroffenheit und Verantwortlichkeit einhergehen und so den Erwerb positiver ökonomischer und ökologischer Einstellungen und auch den Wissenserwerb fördern (Ernst, 1997; Mosler, Ammann \& Gutscher, 1998). Der Identifikationsprozess ermöglicht, persönlich bedeutsame Erfahrungen zu machen, was die Umsetzung von Einstellungen in entsprechendes Verhalten fördert (Fazio \& Zanna, 1981).

\section{ad 3. Kombination von Lernen durch Feedback und} instruierende Erklärungen

In Ergänzung zum Feedback für eigenes Verhalten mittels der Simulationen werden in SimUlme entsprechende Erklärungen zur Verfügung gestellt. Unterhalb der Diagramme werden hierfür jeweils Links zu einer Erklärungs-Website angeboten. Im SimUlme Spiel wird somit Lernen durch direktes Feedback bezüglich der Konsequenzen des eigenen Verhaltens mit Lernen durch Instruktionen und Erklärungen über Systemzusammenhänge kombiniert.

In der pädagogisch-psychologischen Forschung zeigte sich vielfach, dass Feedback in vielen Bereichen eine wirkungsvolle Lernmethode ist, z.B. im Training von Sportlerinnen und Sportlern sowie in der psychologischen oder medizinischen Therapie (Deger, 1994; Legewie \& Nusselt, 1975; Marschall, 1992; Rockmann-Rüger, 1985). Durch Feedback können Informationen und Erfahrungen zugänglich gemacht werden, die sonst nicht der Erfahrung zugänglich sind. Andererseits zeigte sich, dass das Erkennen von Zusammenhängen zwischen unterschiedlichen Parametern in einem virtuellen System auf der ausschliesslichen Grundlage von Feedback mit grossen Problemen verbunden ist. Dies trifft insbesondere in einem komplexen System von Ursache-Wirkungszusammenhängen zu (Reimann, 1990, 1991). Um effektives Lernen zu erreichen, ist hier eine Kombination von Feedback mit erklärenden Instruktionen notwendig. Die Möglichkeit zu einer aktiven Suche von Erklärungen auf Wunsch ist auch motivational sinnvoll. Wenn man in einer Simulation ein Ergebnis erhält, das den Erwartungen widerspricht, entsteht eine kognitive Diskrepanz, welche mit der Motivation für eine zusätzliche Informationssuche einhergeht (Duncker, 1935; Festinger, 1957). Diesem Bedürfnis durch eine Erklärung Rechnung zu tragen, ermöglicht Systemwissen genau dann explizit zu vermitteln, wenn eine Empfänglichkeit hierfür vorhanden ist. Dies sollte den Wissenserwerb und zugleich das Verständnis von Systemzusammenhängen fördern.

\section{ad 4. Verstärkung emotionaler Aspekte durch fotografische Abbildungen}

In SimUlme werden Fotos gezeigt, die korrespondierend zu den Veränderungen einer Grösse wechseln. Zum Beispiel erscheint hinsichtlich artgerechter Nutztierhaltung im Falle einer Verbesserung der Haltungsbedingungen ein Foto mit einer Kuh auf einer Weide im Freiland, im Falle eine Verschlechterung eine Kuh hinter Gittern. Das ergänzende Feedback mit Hilfe von emotional positiven ver- 
sus (vs.) negativen Abbildungen soll die motivationale Grundlage für eine Identifikation mit dem virtuellen System sowie die persönliche Betroffenheit erhöhen. Dies sollte mit einer verstärkten Motivation für den Erwerb von Systemwissen und mit der Förderung positiver Einstellungsveränderungen verbunden sein.

\section{ad 5. Multiple, interaktive Repräsentation von Prozessen und Systemzuständen}

Ein Vorteil computergestützter Lernmedien ist deren Interaktivität. Graphische Darstellungen in interaktiven Lernmedien verändern sich in Folge von Handlungen der Lernenden und können so, als externe Repräsentationen dieser Veränderungen, kognitive Prozesse unterstützen. Lern- bzw. Problemlöseprozesse beinhalten häufig ein Zusammenspiel zwischen internalen (Gedächtnis) und externalen (Wahrnehmung) Repräsentationen (Reimann, 1999). Externale Repräsentationen können hierbei den kognitiven Aufwand für internale Repräsentationen reduzieren und so kognitive Kapazität für andere Aspekte einer Problemlösetätigkeit freisetzen. Eine solche interaktive, externale Repräsentation wird in SimUlme durch die Balkendiagramme bezüglich der acht berücksichtigten Simulationsgrössen erreicht. Diese Diagramme geben der oder dem Spielenden im jeweils aktuellen Spieljahr sowohl einen Überblick über die aktuellen Systemzustände des ökologisch-ökonomischen Gesamtsystems als auch über den Entwikklungsverlauf der acht Systemgrössen in den vorhergehenden Spieljahren. Dies kann den Wissenserwerb und das Verständnis von Systemzusammenhängen fördern, da Veränderungen so sichtbar werden und zum eigenen Verhalten in Bezug gesetzt werden können. Die Erstellung einer solchen, dynamischen externalen Repräsentation wäre ohne Computerunterstützung innerhalb eines für den Unterricht vertretbaren Zeitrahmens nicht möglich. Es ist allerdings zu beachten, dass die durch eine verbesserte externale Repräsentation erreichbare Verminderung kognitiver Beanspruchung nicht generell förderlich ist, da sie im negativen Fall eine oberflächliche Informationsverarbeitung zur Folge haben kann. Es ist daher wichtig, computerbasierende Lernumgebungen im Unterricht in ein geeignetes Instruktionsdesign einzubetten, das Interesse weckt und so eine zentrale Verarbeitung der gelieferten Informationen fördert (Kerres, 2000a; Petty \& Caccioppo, 1986).

\section{Methode}

\section{Stichproben und Experimentelles Design}

Die Anwendung von SimUlme wurde mit Klassen der Sekundarstufe II im Januar 2001 an der Kantonsschule Sargans (KSS) im Kanton St. Gallen getestet. Am Experiment nahmen 12 Schulklassen sowie fünf Lehrkräfte teil, wobei 6 Klassen in der Experimentalbedingung mit Einsatz von SimUlme und 6 Klassen 
in der Vergleichsbedingung ohne SimUlme unterrichtet wurden. Jede Lehrkraft unterrichtete gleich viele Experimentalklassen wie Vergleichsklassen. Die Stichprobengrösse betrug $N=215$, davon 135 Schülerinnen und 80 Schüler. Das mittlere Alter der Schülerinnen und Schüler war $M_{\text {Alter }}=17.6(S D=1.3)$, wobei über $90 \%$ ein Alter von 16 - 19 Jahren hatten. Es bestanden keine signifikanten Unterschiede zwischen den beiden Unterrichtsbedingungen im Hinblick auf das Geschlechterverhältnis und das Alter der Schülerinnen und Schüler.

Der Unterricht für die Experimentalklassen mit SimUlme fand im Informatikzimmer der KSS statt. Dort stand für jede Schülerin und jeden Schüler ein Computer mit Internet-Anschluss zur Verfügung. Der Unterricht der Vergleichsklassen fand in den üblichen Klassenräumen statt. Der Unterricht wurde in beiden Bedingungen entsprechend dem Artikulationsschema von Grell und Grell (1983) in die 5 Phasen Einstimmen/Ausrichten, Informierender Unterrichtseinstieg, Informationsvermittlung, Verarbeiten und Auswerten gegliedert. Als Lernziele wurden Wissenserwerb und der Erwerb positiver umweltbezogener Einstellungen, in beiden Bedingungen gleichermassen festgelegt und spezifiziert. Vor und nach dem Unterricht liessen die Lehrer einen Fragebogen zur Erhebung von auf Verhalten und Werte bezogenen Einstellungen sowie mit zwei Aufgaben zum Umweltwissen ausfüllen. Am Ende des Unterrichts wurden zusätzlich noch Fragen zur subjektiven Bewertung des Unterrichts gestellt. Die Unterrichtsdauer betrug in beiden Bedingungen zwei Schullektionen zu 45 Minuten mit der üblichen Pause von 5 Minuten am Ende der ersten Stunde. Die ersten und die letzten 10 Minuten der Gesamtdauer waren jeweils dem Ausfüllen der experimentellen Fragebögen gewidmet.

Die Vorgaben für die Lehrer für die Phasen (1) Einstimmen/Ausrichten des Unterrichts, (2) Einstieg ins Thema (Umweltfolgen von Lebensmittelkonsum) und (3) Vermittlung grundlegender Informationen waren für beide Unterrichtsbedingungen gleich. Hierbei wurden zu Beginn Folien mit Informationen zum damals in den Medien sehr aktuellen Rinderwahnsinn (BSE) und diverse Zeitungsschlagzeilen und -artikel verwendet, um Interesse am Thema zu wecken. Dann wurde eine Einführung in den Zusammenhang zwischen Lebensmittelkonsum, Produktion und Umweltbelastung gegeben.

Im anschliessenden Unterricht wurde in der Experimentalbedingung SimUlme angewendet. Damit die Schülerinnen und Schüler dieses Spiel bewusst anwenden, erhielten sie ein Protokollblatt, in dem sie für sechs Spieldurchgänge bzw. Einkäufe ihre Zwischenergebnisse und ihre Zielerreichung notierten. Hierbei wurde jeweils eine andere Zielsetzung vorgegeben: 1. realistisch einkaufen, 2. möglichst ökologisch einkaufen, 3. möglichst umweltbelastend einkaufen, 4. Arbeitslosigkeit in der Schweiz minimieren, 5. artgerechte Tierhaltung optimieren sowie 6. freie Wahl der Zielsetzung.

Beim Vergleichsunterricht waren die Lehrer abgesehen vom beschriebenen Einstieg und der Vorgabe des Unterrichtsthemas und der Lernziele, frei in der Wahl ihrer Unterrichtsgestaltung. Nebst lehrerzentriertem Unterricht kamen im 
Vergleichsunterricht im Wesentlichen zwei Unterrichtsinstrumente zur Anwendung: Die Puzzle-Technik nach Frei, Frei-Eiling und Landolt-Marazzi (1990) und das Partner-Interview, für welches den Schülerinnen und Schülern Interview-Vorlagenblätter zum Thema Umweltfolgen von Lebensmitteln bereitgestellt wurden. Für beide Instrumente dienten die Erklärungstexte des SimUlmeSpiels und damit korrespondierende Texte als inhaltliche Orientierung.

\section{Kriterien zur Bewertung des Unterrichts und entsprechende Hypothesen}

Zur Bewertung der Qualität des Unterrichts wurden multiple Kriterien und unterschiedliche Beurteilungsperspektiven genutzt (Kerres, 2000c; Spada \& Reimann, 1982). Die entsprechenden Hypothesen basieren auf den bereits beschriebenen, lernpsychologisch bedeutsamen Aspekten, die bei der Gestaltung von SimUlme berücksichtigt wurden. Die drei Hauptkriterien und die entsprechenden Hypothesen waren:

A. Umweltbezogene Einstellungen (Hypothese A): Die positive Veränderung von Einstellungen ist beim Unterricht mit SimUlme stärker als im Vergleichsunterricht.

B. Wissenserwerb (Hypothese B): Der Wissenserwerb in den Experimentalklassen ist grösser als in den Vergleichsklassen.

C. Subjektive Bewertung des Unterrichts (Hypothese C): Es wurde erwartet, dass der Unterricht mit Anwendung von SimUlme von den Schülerinnen und Schülern und den Lehrern positiver beurteilt wird als der Vergleichsunterricht.

Diese letzte Hypothese basiert auf der spielerischen Lehr-/Lernform, die in Sim Ulme u.a. durch die genannten Möglichkeiten zur Identifikation und Rollenübernahme sowie die emotionale Involvierung der Spieler durch ansprechende Bilder angestrebt wurde. Dies sollte insbesondere mit aktiver Selbstbeteiligung und mit intrinsischem Interesse der Schülerinnen und Schüler am Lernprozess verbunden sein.

\section{Ergebnisse}

\section{Veränderung der Einstellungen}

$\mathrm{Zu}$ Beginn des Unterrichts wurden in beiden Unterrichtsbedingungen neun Fragen zu den Einstellungen der Schülerinnen und Schüler gestellt, die jeweils auf einer fünfstufigen Rating-Skala beantwortet wurden. Die Fragen betrafen die folgenden Themen: 1) Pro-ökologisches Verhalten generell, 2) Bevorzugung regionaler Produkte, 3) Verringerung des Fleischkonsums, 4) Konsum biologisch 
produzierter Fleischprodukte, 5) Konsum biologisch produzierter Gemüseprodukte, 6) Recycling von Abfällen, 7) Bedeutsamkeit des globalen Umweltschutz, 8) Bedeutsamkeit des Tierschutzes und 9) Bedeutsamkeit von Ökonomie bzw. Verringerung der Arbeitslosigkeit.

Zur Vermeidung von Wiederholungseffekten aufgrund identischer Fragestellungen bei der zweiten Messung der Einstellungen am Ende des Unterrichts, wurden zwei verschiedene Fragebogenversionen $(A$ und $B)$ verwendet. Die Reihenfolge (anfangs $A$ und am Ende $B$ vs. anfangs $B$ und am Ende $A$ ) der beiden Versionen war hierbei ausbalanciert, im Hinblick auf die Anzahl von Klassen beider Unterrichtsbedingungen. Die Reihenfolge (2 Stufen: siehe oben) wurde in die entsprechende Varianzanalyse (ANOVA) als unabhängige Variable mit einbezogen, um entsprechende Effekte zu kontrollieren. Neben der Unterrichtsbedingung selbst wurden zusätzlich auch die unabhängigen Variablen Geschlecht und Lehrer sowie die Kovariate Alter in dieser und in den folgenden ANOVAs berücksichtigt. Für eine Darstellung der Ergebnisse in Bezug auf diese zusätzlichen Variablen sei hier auf einen umfangreicheren Forschungsbericht verwiesen (Hansmann, Hesske, Tietje \& Scholz, 2001). Hier wird darauf verzichtet, da diese Ergebnisse keine bedeutsamen Erkenntnisse in Bezug auf die Eignung und Anwendung von SimUlme als Lehr-/Lernmittel oder bezüglich der Gestaltungserfordernisse von Computer-Lernprogrammen generell erbrachten.

Zur Untersuchung der Einstellungsveränderungen wurde eine ANOVA mit den genannten unabhängigen Variablen und dem Messwiederholungsfaktor $U n$ terricht (2 Stufen: Messung am Anfang vs. am Ende des Unterrichts) durchgeführt, auf Basis der Mittelwerte der Schülerinnen und Schüler bei den neun Einstellungsfragen. ${ }^{1}$ Gemittelt über beide Unterrichtsbedingungen kam es zu einer Verbesserung der mittleren Einschätzungen von $M_{\text {Anf }}=3.66 \mathrm{zu}$ Beginn des Unterrichts auf $M_{\text {Ende }}=4.05$ am Ende des Unterrichts, was allerdings nicht signifikant war $(p=.71)$. Abbildung 1 zeigt die Veränderungen der mittleren Einschätzungen der Schülerinnen und Schüler bei den einstellungsbezogenen Fragen getrennt für die beiden Unterrichtsbedingungen. Wie in Abbildung 1 zu sehen ist, ergaben sich tendenzielle Verbesserungen in den Einstellungen durch den Umweltunterricht vor allem bei den vier verhaltensbezogenen Fragen 2-5, die den Bereich Ernährung betrafen und somit im Unterricht direkt thematisiert wurden. Die Verbesserungen der Einstellungen in der Unterrichtsbedingung mit SimUlme waren stärker ausgeprägt als in der Vergleichsbedingung. In der Bedingung mit SimUlme stiegen die Einschätzungen von $M_{\text {Anf }}=3.68$ auf $M_{\text {Ende }}=$ $4.12(\varnothing=0.44)$, während der Anstieg in der Bedingung ohne SimUlme von $M_{\text {Anf }}$ $=3.63$ auf $M_{\text {Ende }}=3.96(\varnothing=0.33)$ erfolgte. Der entsprechende Wechselwirkungseffekt war beim zweiseitigen statistischen Test knapp nicht signifikant, $F(1,189)=3.64, p=.058$. Entsprechend der einseitig formulierten Hypothese $A$ spricht dieses Ergebnis nach Ansicht der Autoren dennoch eher für eine grössere positive einstellungsverändernde Wirkung des durch SimUlme unterstützten Unterrichts, im Vergleich zum herkömmlichen Umweltunterricht. 


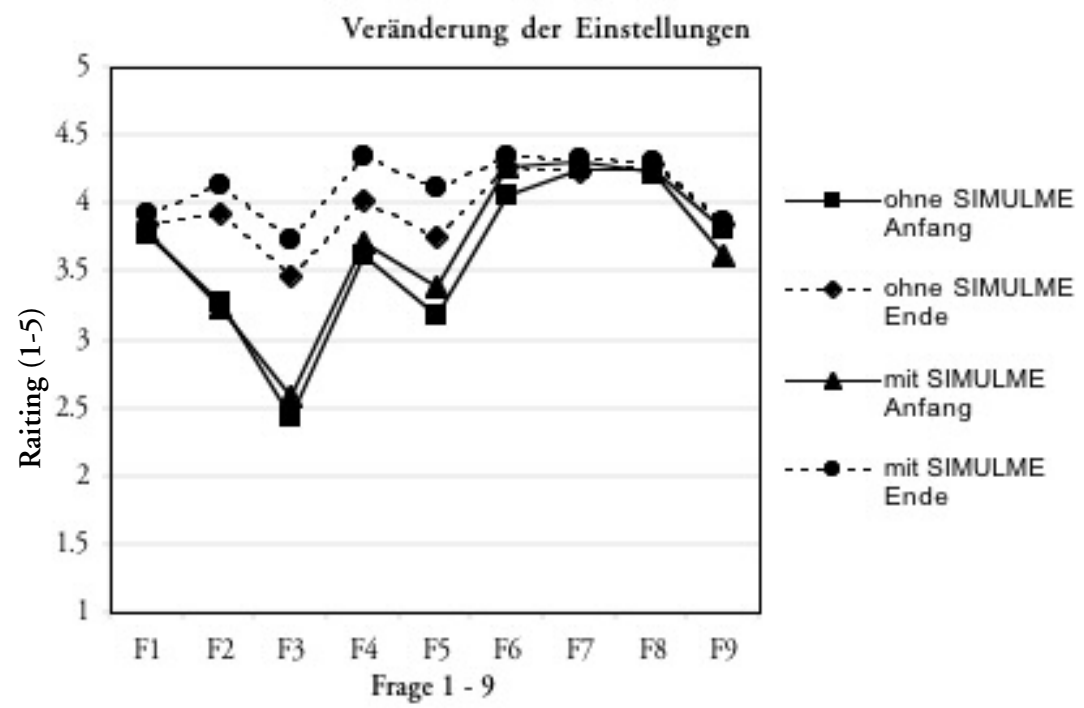

Unterrichtsbedingungen mit SimUlme vs. ohne SimUlme; Anfang = zu Beginn des Unterrichts, Ende = am Ende des Unterrichts; $1=$ negativ $/$ minimal, $5=$ positiv $/ \mathrm{maximal}$.

Fragen 1-9: F1_Umweltverhalten generell, F2_Regionale Lebensmittel bevorzugen, F3_Fleisch-konsum verringern, F4_Tierprodukte aus biologisch orientierter Produktion bevorzugen, F5_Gemüse aus biologisch orientierter Produktion bevorzugen, F6_beim Recycling von Abfällen mitmachen, F7_Bedeutsamkeit-Umweltschutz, F8_BedeutsamkeitTierschutz, F9_Bedeutsam-keit-Wirtschaftsentwicklung/ Bekämpfung der Arbeitslosigkeit.

Abbildung 1: Veränderung der Einstellungen der Schülerinnen und Schüler in den Unterrichtsbedingungen mit SimUlme vs. ohne SimUlme.

\section{Analyse des Wissenserwerbs}

Zur Untersuchung des Wissensgewinns wurden zwei Aufgaben, die jeweils aus 10 Teilfragen bestanden, zu Beginn und dann nochmals am Ende des Unterrichts vorgegeben. In Aufgabe 1 sollten die Schülerinnen und Schüler das Ausmass der Umweltbelastung durch 10 spezifische Aktivitäten der Nahrungsmittelwirtschaft bewerten. Die Aktivitäten waren unterschiedliche Teile des Produktkreislaufs der betrachteten Lebensmittel, nämlich die Produktion von Gemüse und Fleisch bei unterschiedlichen Produktionsweisen sowie der Transport dieser Lebensmittel bei unterschiedlicher Herkunft und verschiedenen Transportmitteln. Bei Aufgabe 2 sollten in einer Liste von 10 Variablen alle Variablen gekennzeichnet werden, welche durch die Einkaufsentscheidungen der Schweizer Konsumenten und Konsumentinnen bei Gemüse und Fleisch beeinflusst werden (z.B. Arbeitslosigkeit in der Schweiz, Verwirklichung artgerechter Tierhaltung, Einkommen der Landwirte etc.). Aufgabe 1 erfasste somit den Er- 
werb von quantitativem, umweltbezogenem Wissen, während Aufgabe 2 in stärkerem Masse qualitatives Systemverständnis erfragte. Der Wissenserwerb der Schülerinnen und Schüler bezüglich der beiden Aufgaben wurde durch eine ANOVA mit den zwei Messwiederholungsfaktoren Unterricht (2 Stufen: Anfang vs. Ende) und Aufgabe (2 Stufen: Aufgabe 1 vs. 2) analysiert. Die ANOVA beinhaltete als abhängige Variable für beide Aufgaben den Anteil korrekt gelöster Teilfragen $\left(A_{\mathrm{kor}}\right)$.

Tendenziell zeigte sich im Mittel über beide Unterrichtsbedingungen und Aufgaben eine Verbesserung des Umweltwissens von $A_{\mathrm{kor}}=0.46 \mathrm{zu}$ Beginn auf $A_{\mathrm{kor}}$ $=0.63(\varnothing=0.17)$ am Ende des Unterrichts, was allerdings nicht signifikant war $(p=.19)$. Vielmehr kam es zu einem deutlich signifikanten Wechselwirkungseffekt Unterricht $x$ Aufgabe $x$ Unterrichtsbedingung, $F(1,188)=9.29, p<.01$. Abbildung 2 zeigt, dass bezüglich Aufgabe 1 der Zugewinn an Wissen in der Unterrichtsbedingung ohne SimUlme stärker war als in der Bedingung mit SimUlme, während bei Aufgabe 2 genau das Gegenteil der Fall war. Hier war die Verbesserung des Umweltwissens in der Bedingung mit SimUlme stärker ausgeprägt als im Vergleichsunterricht. Hypothese B, die einen generell besseren Wissenserwerb in der Bedingung mit SimUlme beinhaltete, wurde somit von den Daten nicht bestätigt.

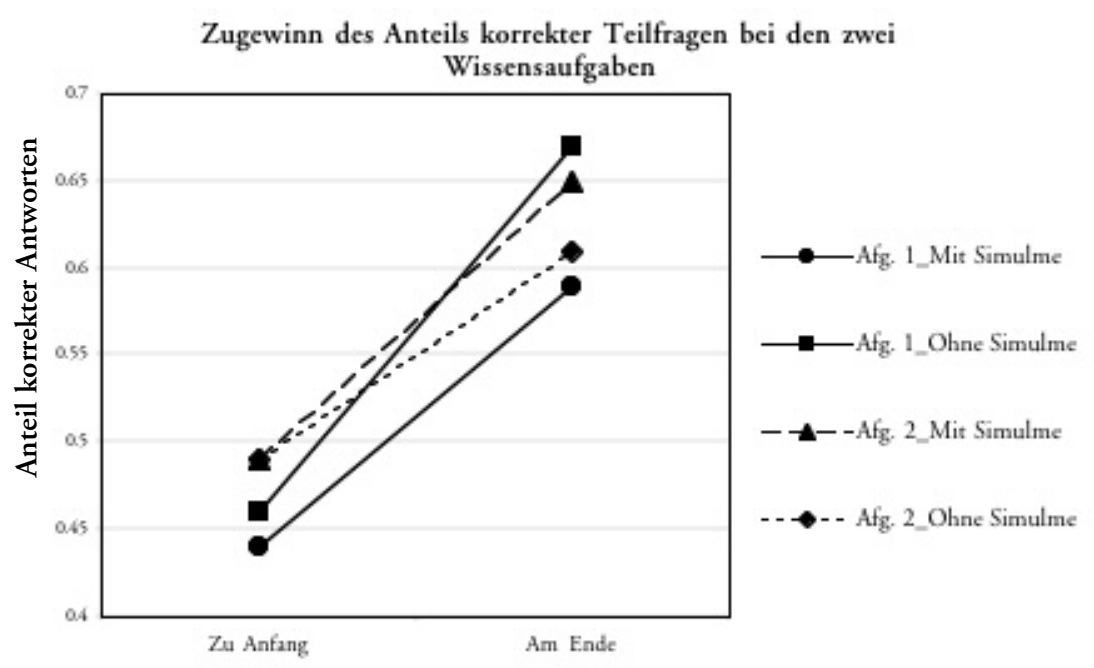

Afg. 1 = Aufgabe 1, Einschätzung der Umweltbelastung von 10 wirtschaftlichen Aktivitäten im Produktkreislauf von Gemüse und Fleisch. Afg. 2 = Aufgabe 2, Auswahl von Grössen, welche durch Einkaufsentscheidungen bei Gemüse und Fleisch beeinflusst werden; Zu Anfang vs. am Ende = Bei Beginn vs. am Ende des Unterrichts.

Abbildung 2: Veränderung des Anteils korrekter Teilfragen bei den Wissensaufgaben 1 und 2 in der Unterrichtsbedingung mit vs. ohne SimUlme. 


\section{Analyse der subjektiven Bewertungen des Unterrichts}

Die Schülerinnen und Schüler bewerteten den Unterricht in beiden experimentellen Bedingungen positiv. Wie Tabelle 1 zeigt, weichen die mittleren Bewertungen für alle acht abgefragten Kriterien signifikant positiv von der neutral formulierten, mittleren Stufe der 5-stufigen Rating-Skala ab (immer, $p<.001$ ).

Zwischen den Bedingungen mit vs. ohne ergaben sich signifikante Unterschiede bezüglich der Bewertung der Interessantheit des Unterrichts, bezüglich des Ausmasses aktiver Eigenbeteiligung am Unterricht und im Hinblick auf die Verständlichkeit der Vermittlung der Lerninhalte. Die Schülerinnen und Schüler fanden den Unterricht mit SimUlme signifikant interessanter als ohne SimUlme, $F(1,185)=4.23, p<.05$. Sie konnten ihrer eigenen Einschätzung nach im Unterricht mit SimUlme stärker selbst aktiv werden, $F(1,185)=8.44, p<.001$, und die Vermittlung der Lerninhalte wurde mit SimUlme als verständlicher beurteilt, $F(1,185)=3.71, p<.05$ (vgl. Tabelle 1$)$.

\section{Tabelle 1: Analyse der Einschätzungen der Schülerinnen und Schüler bei den abschliessenden Fragen zur Bewertung des Unterrichts}

\begin{tabular}{|l|cc|c|c|}
\hline $\begin{array}{c}\text { Einschätzungen der } \\
\text { Schülerinnen und Schüler } \\
\text { zu den Abschlussfragen }\end{array}$ & $\begin{array}{c}\text { Mittelwerte } \\
\text { Bedingung } \\
\text { - SimUlme - }\end{array}$ & $\begin{array}{c}\text { Signifikanz } \\
\text { der Differenz }\end{array}$ & $\begin{array}{c}\text { Signifikanz der Abweichung } \\
\text { vom neutralen } \\
\text { Skalenpunkt 3.0 }\end{array}$ \\
\hline Interessantheit des Unterrichts & 3.50 & 3.74 & Mit vs. Ohne & Gesamtmittelwert \\
\hline Aktive eigene Beteiligung & 3.51 & 3.89 & $\mathrm{p}<.05$ & $\mathrm{p}<.001$ \\
\hline Neues gelernt & 3.48 & 3.42 & $\mathrm{n}$. s. & $\mathrm{p}<.001$ \\
\hline Wichtigkeit des Wissens & 3.90 & 3.89 & n.s. & $\mathrm{p}<.001$ \\
\hline Anwendbarkeit im Alltag & 4.36 & 4.19 & n.s. & $\mathrm{p}<.001$ \\
\hline Wissen übertragbar & 3.83 & 3.81 & $\mathrm{n} . \mathrm{s}$. & $\mathrm{p}<.001$ \\
\hline Verständlichkeit des Unterrichts & 3.58 & 3.96 & $\mathrm{p}<.05$ & $\mathrm{p}<.001$ \\
\hline Lust auf mehr Umweltunterricht & 3.40 & 3.33 & n.s. & $\mathrm{p}<.001$ \\
\hline
\end{tabular}

Anmerkung: $n . s .=$ nicht signifikant; Die Einschätzungen erfolgten auf einer 5-stufigen RatingSkale von 1 = sehr negativ bis 5 = sehr positiv).

a Auf eine Bonferroni Korrektur zur Absicherung gegen zufällige Signifikanzen bei mehreren unabhängigen t-tests (Rosenthal \& Rosnow, 1991) wurde hier verzichtet, da eine Varianzanalyse mit Messwiederholung über alle acht Fragen einen hoch signifikanten Wechselwirkungseffekt Unterrichtsbedingung $x$ Frage ergab, $F(6.38,1326.04)=4.32$, $p<.001$ (Korrektur der df nach Huynh-Feldt, ( =.91).

Die Lehrer beurteilten den Unterricht im Mittel ebenfalls in beiden Unterrichtsbedingungen positiv (Signifikanz des Abstands zum neutralen Mittelwert $M=3.0$ der Skala, bei allen acht Fragen, $p<.01$ ). Bei den einzelnen Fragen ergaben sich keine signifikanten Unterschiede zwischen den beiden Unterrichtsbedingungen, obgleich die Bewertung von SimUlme im Hinblick auf jedes der acht Kriterien tendenziell besser war als die des Unterrichts ohne SimUlme. Ergänzend wurde eine gemeinsame ANOVA bezüglich aller acht Fragen gerech- 
net, um Unterschiede in der mittleren Beurteilung über die acht Fragen hinweg zu analysieren. Die entsprechende ANOVA enthielt die beiden Messwiederholungsvariablen Unterrichtsbedingung (2 Stufen) und Frage (8 Stufen). Hier ergab sich zwar ebenfalls kein signifikanter Unterschied zwischen den Bewertungen des Unterrichts mit vs. ohne SimUlme durch die Lehrer $\left(M_{\mathrm{Mit}}=4.29\right.$ vs. $M_{\text {Ohne }}$ = 4.07), $F(1,5)=4.42$, $p=.089$. Die positive Tendenz der Mittelwertsunterschiede zugunsten von Unterricht mit SimUlme erscheint jedoch durchaus erwähnenswert.

\section{Diskussion}

Sowohl die Schülerinnen und Schüler als auch die Lehrer bewerteten den Umweltunterricht in beiden Unterrichtsbedingungen hinsichtlich aller erhobenen Aspekte als positiv. Das Lernspiel SimUlme wurde somit als ein innovatives Lehr-/Lernmittel gut akzeptiert.

Obwohl im vorliegenden Experiment für die Schülerinnen und Schüler kein Leistungsdruck zur Erreichung der Lernziele bestand, wurde in beiden Unterrichtsmodi Umweltwissen erfolgreich vermittelt. Beim Unterricht mit SimUlme fand der nachgewiesene Wissensgewinn allerdings hinsichtlich anderer Aspekte statt als beim Unterricht ohne SimUlme. Die Charakteristika der beiden verwendeten Aufgabentypen zum Umweltwissen deuten im Zusammenhang mit den Ergebnissen darauf hin, dass SimUlme verstärkt Systemverständnis fördert, während quantitatives Wissen stärker im Vergleichsunterricht gefördert wurde. Dies könnte auf den Vorteil von SimUlme zurückzuführen sein, eine anschauliche Repräsentation von Systemzuständen und Entwicklungen zu liefern, die ohne das Spiel im Unterricht kaum möglich ist. Ein kognitiver Vorteil der anschaulichen, externalen Repräsentation der Konsequenzen der eigenen Einkaufsentscheidungen durch die Computersimulationen kann in der Freisetzung internaler, kognitiver Kapazitäten für die Erforschung der Ursachen dieser Entwicklungen bestehen, was zu einem verbesserten Systemverständnis beitragen kann. Der bessere Erwerb des quantitativen Wissens im Vergleichsunterricht könnte andererseits mit einer Ablenkung durch eben diese Repräsentationen zusammenhängen. Aufgrund der vorliegenden Ergebnisse lässt sich somit nicht aussagen, ob Unterricht mit oder ohne SimUlme einen grösseren Wissenserwerb verspricht. Vielmehr legen die Ergebnisse nahe, dass sich die Lernprozesse und Lerninhalte in umweltbezogenem Unterricht mit und ohne SimUlme komplementär ergänzen. Insbesondere bei der Umweltbildung erscheint es ohnehin wichtig, auch gegenständliche, praktische Projekte durchzuführen und neben medialen Präsentationen mittels Monitorbildern und (Buch-)Texten auch Erfahrungen mit konkreten Objekten und Umwelten zu vermitteln (Bauer, 1995). Eine Beschränkung von (Umwelt-)Unterricht auf die Interaktion zwischen Schülern und Computern ist nicht zweckmässig. 
In Bezug auf das Ziel einer Einstellungsveränderung in pro-ökologische Richtung erreichte der Unterricht mit SimUlme etwas stärkere Effekte als der Vergleichsunterricht. Mögliche Verhaltensänderungen der Schülerinnen und Schüler als Folge des Unterrichts konnten im Rahmen der vorliegenden Studie aufgrund praktischer Beschränkungen leider nicht erfasst werden. Dies stellt jedoch eine bedeutsame Fragestellung dar, die in einer zukünftigen Studie untersucht werden sollte. Prognosen hierfür müssen vor dem Hintergrund des vorliegenden psychologischen Wissens über den häufig geringen Zusammenhang zwischen Umwelteinstellungen und Umweltverhalten generell vorsichtig ausfallen (Diekmann \& Preisendörfer, 1992; Schahn, 1993). Auch der Erwerb von Umweltwissen kann eine Veränderung des Verhaltens der Schülerinnen und Schüler in Richtung auf pro-ökologisches Verhalten bewirken (Fietkau \& Kessel, 1981). Die Verbesserungen bezüglich Umweltwissen und Umwelteinstellungen in der Unterrichtsbedingung mit SimUlme deuten somit immerhin auf die Möglichkeit einer ökologischen Verbesserung des künftigen Ernährungsverhaltens der Schülerinnen und Schüler in der Experimentalbedingung hin. Als äusseren Hindernisgrund für ein intendiertes, ökologischeres Verhalten erwähnten allerdings mehrere Schülerinnen, dass zu Hause nicht sie selber, sondern jeweils ihre Eltern die Lebensmitteleinkäufe tätigen würden.

Den Autoren erscheint Edutainment als eine vielversprechende Gestaltungsform von computerisierten Lernprogrammen in der Schule, da spielerisches Lernen als eine motivierende und daher effektive Lernform angesehen werden kann. Eine weitere Fragestellung der Untersuchung war daher zu analysieren, inwieweit SimUlme tatsächlich ein spielerisches Lernen ermöglicht, ob also der hier verwendete Begriff Lernspiel zutrifft. Bei den prozessorientierten Fragen am Ende des Unterrichts gaben die Schülerinnen und Schüler in der SimUlme Unterrichtsbedingung in signifikant stärkerem Masse an, dass der Unterricht interessant war und dass sie sich aktiv selbst beteiligen konnten. Dies belegt, dass Sim Ulme eine spielerische Form des Lernens erreicht. Die interressierte, aktive Selbstbeteiligung deutet auf kreatives, entdeckendes und selbstgesteuertes Lernen hin, was einen Schritt weg vom eher rezeptiven Lernen im Frontalunterricht hin zu spielerischem Lernen bedeutet (Bauer, 1995). Ein didaktischer Mehrwert des Lernprogramms könnte daher in Motivationsgewinnen für das Lernen bestehen. In diesem Zusammenhang ist jedoch auch zu berücksichtigen, dass Neuheit und Attraktivität des Lernmediums Internet einen positiven Einfluss auf die subjektiven Bewertungen und auf das Engagement der Schülerinnnen und Schüler in der Unterrichtsbedingung mit SimUlme gehabt haben könnten. Dies kann auf Grundlage des vorliegenden Experiments nicht ausgeschlossen werden.

Die gute Bewertung der Verständlichkeit des Unterrichts mit SimUlme spricht dafür, dass die begleitenden Erklärungen zu den Simulationen des Sim Ulme-Spiels den Schülerinnen und Schülern eine gute Orientierung geben, die im Rahmen des entdeckenden Lernens ein Übermass an blindem Trial and error Problemlösen vermeidet. In diesem Zusammenhang ist auch das Alter der Schü- 
lerinnen und Schüler zu berücksichtigen. Beim vorliegenden Experiment lag die Altersspanne zwischen 16 und 19 Jahren. Die hohe Verständlichkeit des Unterrichts für die Schülerinnen und Schüler dieses Alters deutet darauf hin, dass auch etwas jüngere Altersstufen von SimUlme profitieren könnten. Es liegen hierzu jedoch bislang noch keine Ergebnisse oder Erfahrungsberichte vor.

Eine bedeutsame Fragestellung im Zusammenhang mit der Evaluation der Anwendung von SimUlme ist die Generalisierbarkeit der Ergebnisse in Bezug auf den Einsatz von Computer-Lernprogrammen in der Schule generell. Für entsprechende Schlussfolgerungen müssen die Besonderheiten von SimUlme berücksichtigt werden. Hier ist zum einen die Thematisierung des Umweltschutzes durch das Spiel zu erwähnen. Das Interesse der Schüler für dieses Thema könnte möglicherweise zum Engagement der Schüler bei der Beschäftigung mit SimUlme beigetragen haben. Allerdings wurde in der Vergleichsbedingung Unterricht zum gleichen Thema durchgeführt. Es erscheint daher aufgrund der gefundenen Unterschiede plausibel, dass bei anderen Themenbereichen vergleichbare positive Effekte durch Computer-Lernspiele erreicht werden können. Des weiteren wurden bei der Gestaltung von SimUlme einige lernpsychologisch relevante Gesichtspunkte berücksichtigt, die, wie dargestellt wurde, positive pädagogisch-psychologische Effekte haben können. Der mögliche Einfluss einiger dieser Aspekte wurde in der bisherigen Diskussion bereits im Zusammenhang mit den vorliegenden Ergebnissen erörtert. Aufgrund der gemeinsamen Wirkung aller dieser Aspekte im Unterricht mit SimUlme ist jedoch eine exakte Zurückführung der Effekte von SimUlme auf die einzelnen Wirkfaktoren nicht möglich. Bei der Auswahl und Entwicklung von computerisierten Lernprogrammen für den Schulunterricht sollten, soweit dies im speziellen Fall passend erscheint, möglichst viele der dargestellten Gestaltungsaspekte verwirklicht werden. Diese beinhalten, wie beschrieben wurde: Die Möglichkeit, sich mit einer bedeutsamen Rolle identifizieren zu können, eine Kombination von Ergebnis-Feedback mit instruierenden Erklärungen, eine Förderung emotionalen Erlebens beim Lernen und eine multiple und interaktive Repräsentation von Prozessen und Systemzuständen. Hinsichtlich dieser Aspekte besteht im übrigen auch bei SimUlme durchaus noch ein Bedarf bzw. ein Potential zu weiteren Verbesserungen. Die Ergebnisse des durchgeführten Experimentes legen nahe, dass im Hinblick auf Umwelteinstellungen und Umweltwissen durch eine Verbesserung des computerisierten Lernprogramms noch stärkere Lerneffekte erreichbar wären. Eine solche Weiterentwicklung des SimUlme-Lernspiels soll auf Grundlage des vorliegenden Experiments und des zahlreich erhaltenen Feedbacks zum Spiel auch erreicht werden. Denkbar sind hierbei unter anderem (a) der Einbau auditiver Elemente, um den Wissenserwerb durch die Informationsaufnahme über verschiedene Sinneskanäle zu fördern, (b) eine attraktivere Einbindung der Erklärungsseiten in den Spielverlauf, verbunden mit einer verbesserten, auch graphischen Darstellung der Erklärungen, um das Aufsuchen und die Nutzung dieses für den Wissenserwerb zentralen Angebots zu fördern 
und (c), ähnlich zu Ereigniskarten in bekannten Gesellschaftsspielen, die Integration von visualisierten Ereignissen, deren Wahrscheinlichkeit vom Spielver-halten abhängt, um ein zusätzliches Element der Spannung zu erreichen. Die Anreicherung des Spiels um Elemente politischer Entscheidungsprozesse oder um Aspekte der Zusammenhänge zwischen Ernährungsverhalten und Gesundheit sind zusätzliche, inhaltliche Ansatzpunkte für eine Weiterentwicklung des Spiels.

Informationen zur Durchführung von Umweltunterricht mit SimUlme und entsprechende Arbeitsmaterialien stehen unter der Webadresse http://andros.ethz.ch Iks/Info_ks.asp zur Verfügung. Diese ergänzenden Arbeitsmaterialien können genutzt werden, um die Anwendung von SimUlme in ein umfassendes Instruktionsdesign einzubetten. Eine entsprechende sorgfältige Einbettung ist generell wichtig für die erfolgreiche Durchführung von computer-unterstütztem Unterricht (Kerres \& Jechle, 2000; Kerres, 2001). In Ergänzung zu dem hier gewählten Vorgehen könnte man hierbei auch versuchen, Übungen im Rahmen von Gruppenarbeit durchzuführen. Konzepte der Gruppenarbeit können Motivationsgewinne erreichen und sind darüber hinaus mit aktiver, realer zwischenmenschlicher Kommunikation verbunden. Letzteres erscheint den Autoren insbesondere auch beim computerunterstützten Lernen wichtig.

\section{Anmerkungen}

Der Schulleitung der Kantonsschule Sargans (KSS), dem Informatikbeauftragten der KSS, Joseph Vogel, und den am Experiment beteiligten Lehrkräften, Stefan Castelberg, Hans Conrad, Thomas Flüeler, Jens Listemann und Stefan Hesske danken wir für ihre Unterstützung. Dem Schweizerischen Nationalfonds zur Förderung der wissenschaftlichen Forschung (SNF) danken wir für die finanzielle Trägerschaft der Entwicklung des SimUlme Spiels und zur Durchführung der vorliegenden Forschungsarbeit. Die Arbeit wurde im Rahmen des Teilprojekts VIII «Lebensstile, Konsummuster und Ökologische Folgen» des Integrierten Projekts Gesellschaft (IPG) des Schwerpunktprogramms Umwelt (SPPU) des SNF durchgeführt.

\section{Fussnoten}

1 Die Variable Schulklasse konnte in den Analysen nicht berücksichtigt werden, da eine bestimmte Ausprägung der Variable Schulklasse immer mit einer bestimmten Unterrichtsbedingung einhergeht. Die Prozesse innerhalb einer Klasse verlaufen nicht statistisch unabhängig voneinander. Streng genommen sind daher Analysen auf Basis der Klassenmittelwerte mit den einzelnen Klassen als Analyseeinheit notwendig. Dies war hier aufgrund der Anzahl von nur 6 Schulklassen je Unterrichtsbedingung nicht praktikabel. Eine gewisse Berechtigung für das gewählte Vorgehen ergibt sich aus der Berücksichtigung der Variable Lehrer in den Analysen. Ein Teil der auf bestimmte Schulklassen und entsprechende Unterrichtsverläufe zurückgehenden gemeinsamen Varianzen mehrerer Schülerinnen und Schüler ist in den vorliegenden Analysen durch die Variable Lehrer berücksichtigt. Diese beinhaltet in den vorliegenden Analysen nicht nur die Wirkungen eines be-stimmten Lehrerverhaltens, sondern auch Effekte, die auf die Schulklassen eines Lehrers zurückzuführen sind (insbesondere im Rahmen der Wechselwirkung Lehrer $x$ Unterrichtsbedingung). 


\section{Bibliographie}

Bandura, A. (1979). Sozial-kognitive Lerntheorie. Stuttgart: Klett-Cotta.

Bauer, W. (1995). Multimedia in der Schule? In L. J. Issing \& P. Klimsa (Hrsg.), Information und Lernen mit Multimedia. Berlin: Beltz.

Deger, K. (1994). Sprechmotorisches Lernen mit Feedback: Grundlagen und therapeutische Anwendung. Pfaffenweiler: Centaurus-Verlagsgesellschaft.

Diekmann, A. \& Preisendörfer P. (1992). Persönliches Umweltverhalten. Diskrepanzen zwischen Anspruch und Wirklichkeit. Kölner Zeitschrift für Sozialpsychologie, 44, (2), 226251.

Duncker, K. (1935). Zur Psychologie des produktiven Denkens. Berlin: Springer.

Ernst, A. (1997). Ökologisch-soziale Dilemmata: Psychologische Wirkmechanismen des Umweltverhaltens. Weinheim: Beltz.

Fazio, R. H. \& Zanna, M. P. (1981). Direct experience and attidude-behavior consistency. In L. Berkowitz (Hrsg.), Advances in Experimental Social Psychology (S. 161-202). New York: Academic Press.

Festinger, L. (1957). A theory of cognitive dissonance. Evanston: Row, Patterson and Company. Fietkau, H.-J. \& Kessel, H. (1981). Umweltlernen. Königstein/Taunus: Hain.

Frei, K., Frei-Eiling, A. \& Landolt-Marazzi, E. (1990). Allgemeine Didaktik. Zürich: ETH Zürich, Institut für Verhaltenswissenschaften, Verlag der Fachvereine.

Glowalla, U. \& Häfele, G. (1995). Einsatz elektronischer Medien: Befunde, Probleme und Perspektiven. In L. J. Issing \& P. Klimsa (Hrsg.), Information und Lernen mit Multimedia. Berlin: Beltz.

Grell, J. \& Grell, M. (1983). Unterrichtsrezepte. Weinheim: Beltz.

Hansmann, R. (2001). Die INFO-Intervention (Improvement of Normative Functioning and Output of Groups) zur Verbesserung von Gruppenentscheidungen - Eine experimentelle Analyse kollektiver Problemlöseprozesse. In R. W. Scholz \& H. W. Crott, Psychologie des Entscheidungsverhaltens und Konfliktes, Vol. 9. Frankfurt: Peter Lang.

Hansmann, R., Hesske, S., Tietje, O. \& Scholz, R. W. (2001). Internet-unterstützte Umweltbildung: Eine experimentelle Studie zur Anwendung des Online-Simulationsspiels Sim Ulme im Schulunterricht (UNS Working Paper, 26). Zürich: ETH Zürich, Umweltnaturund Umweltsozialwissenschaften.

Hassenstein, B. (2001). Verhaltensbiologie des Kindes (5. Aufl.). Heidelberg: Spektrum-Verlag.

Hesske, S. \& Frischknecht-Tobler, U. (1999). Transdisziplinäre Umweltbildung «Boden und Schwermetalle» - Ein Beitrag zur nachhaltigen Bodennutzung. Forschungsbericht. Zürich: Umweltnatur- und Umweltsozialwissenschaften (UNS), Eidgenössische Technische Hochschule Zürich.

Hesske, S. \& Frischknecht-Tobler, U. (2000). «Transdisciplinary environmental education. Example». Soil and heavy Metals. In: R. Häberli, R. W. Scholz, A. Bill \& M. Welti (Hrsg.), Proceedings of the International Transdisciplinarity 2000 Conference. "Transdisciplinarity: Joint Problem-Solving among Science, Technology and Society. Workbook I: Dialogue Sessions and Idea Market» (S. 652-655). Zürich: Eidgenössische Technische Hochschule Zürich.

Jungbluth, N. (2000). Umweltfolgen des Nahrungsmittelkonsums: Beurteilung von Produktmerkmalen auf Grundlage einer modularen Ökobilanz. Berlin: dissertation.de.

Kaufmann-Hayoz, R. \& Di Giulio, A. (Hrsg.) (1996). Umweltproblem Mensch: Humanwissenschaftliche Zugänge zu umweltverantwortlichem Handeln. Bern: Paul Haupt.

Kerres, M. (2000a). Internet und Schule. Eine Übersicht zu Theorie und Praxis des Internets in der Schule. Zeitschrift für Pädagogik, 46, (1), 113-130.

Kerres, M. (2000b). Potenziale des Lernens im Internet: Fiktion oder Wirklichkeit? In: H. Hoffmann (Hrsg.), Deutsch global? Neue Medien, eine Herausforderung für die deutsche Sprache (S. 170-195). Köln: Du Mont. 
Kerres, M. (2000c). Information und Kommunikation beim mediengestützten Lernen. Entwicklungslinien und Perspektiven mediendidaktischer Forschung. Zeitschrift für Erziehungswissenschaft, 3, (1), 111-130.

Kerres, M. (2001). Multimediale und telemediale Lernumgebungen. Konzeption und Entwicklung. 2. überarbeitete und erweiterte Auflage. München: R. Oldenbourg Verlag.

Kerres, M. \& Jechle, Th. (2000). Betreuung des Lernens in telemedialen Lernumgebungen. Unterrichtswissenschaft, 28, (3), 257-277.

Kyburz-Graber, R., Rigendinger, L., Hirsch Hadorn, G. \& Werner Zentner, K. (1997). Sozioökologische Umweltbildung. Hamburg: Krämer.

Kyburz-Graber, R. \& Schlüter, K. (2001). Umweltbildung im 20. Jahrhundert: Anfänge, Gegenwartsprobleme, Perspektiven. Münster: Waxmann.

Legewie, H. \& Nusselt, L. (1975). Biofeedback-Therapie: Lernmethoden in der Psychosomatik, Neurologie und Rehabilitation. München, Berlin \& Wien: Urban \& Schwarzenberger.

Marschall, F. (1992). Informationsfrequenz und motorisches Lernen: Zur Frage von Häufigkeit und Verteilung ergänzender Feedback-Information bei sportmotorischen Optimierungsprozessen. Frankfurt am Main: Peter Lang.

Moscovicci, S. \& Faucheux, C. (1972). Social influence, conformity bias and the study of active minorities. In L. Berkowitz (Hrsg.), Advances in Experimental Social Psychology (S. 140-202). New York: Academic Press.

Mosler, H. J., Ammann, F. \& Gutscher, H. (1998). Simulation des Elaboration Likelihood Model (ELM): Ein Beispiel zur Entwicklung und Analyse von Umweltinterventionen. Bern: Hans Huber.

Mugny, G. (1982). The power of minorities. London: Academic Press.

Petty, R. E. \& Caccioppo, J. T. (1986). The Elaboration Likelihood Model of persuasion. In L. Berkowitz (Hrsg.), Advances in Experimental Social Psychology, (S. 161-202). New York: Academic Press.

Reigeluth, Ch. M. (1983). Instructional design: What is it and why is it? In Ch. M. Reigeluth (Hrsg.), Instructional theories and models: An overview of their current status (S. 3-36). Hillsdale: Lawrence Erlbaum.

Reimann, P. (1990). Problem solving models of scientific discovery learning processes. Frankfurt: Peter Lang.

Reimann, P. (1991). Detecting functional relations in a computerized discovery environment. Learning and Instruction, 1, 45-65.

Reimann, P. (1999). The role of external representations in distributed problem solving. Learning and Instruction, 9, 411-418.

Rockmann-Rüger, U. (1985). Zum Einfluss der Zeitstruktur der Feedback-Vermehrung auf sensomotorische Optimierungsprozesse im Sport. Berlin: Copy-Center in Dahlem.

Rosenthal, R. \& Rosnow, R. L. (1991). Essentials of Behavioral Research: Methods and Data Analysis. New York: McGraw-Hill.

Schahn, J. (1993). Die Kluft zwischen Einstellung und Verhalten beim individuellen Umweltschutz. In J. Schahn \& T. Giesinger (Hrsg.), Psychologie für den Umweltschutz (S. 2949). Weinheim: Psychologie Verlags Union.

Spada, H. \& Reimann, P. (1982). Educational Measurement. The German Journal of Psycho$\log y, 6,(4), 286-305$. 


\section{Formation écologique basée sur l'internet: étude expérimentale sur l'application du jeu internet SimUlme à I'école}

\section{Résumé}

Un jeu internet a été développé pour thématiser et visualiser de manière ludique les conséquences économiques et écologiques de la consommation alimentaire. Dans l'expérience, 12 classes scolaires ont éte enseignées sur ces conséquences, 6 classes faisant usage du jeu SimUlme (condition expérimentale), 6 classes sans utiliser ce jeu (condition de comparaison). La compréhension, l'autoparticipation active et l'intérêt pendant l'enseignement étaient jugés significativement plus positifs par les élèves dans la condition expérimentale que dans la condition de comparaison. Dans la tendance, l'enseignement avec SimUlme a amélioré plus fortement les attitudes écologiques concernant l'alimentation. Le savoir écologique a été stimulé de manière complémentaire dans les deux conditions. Les résultats soutiennent l'application des jeux adéquats basés sur l'internet dans l'enseignement à l'école.

\section{Formazione ecologica basata su internet. Studio sperimen- tale relativo all'applicazione del gioco internet SimUlme a scuola}

\section{Riassunto}

Per tematizzare e visualizzare in modo ludico le conseguenze economiche ed ecologiche dei consumi alimentari è stato sviluppato un gioco internet. Nella sperimentazione sono state coinvolte 12 classi, 6 sperimentali con applicazione e 6 di controllo senza applicazione del gioco. Gli allievi delle classi sperimentali hanno giudicato la comprensione, l'interesse e la possibilità di partecipazione in maniera significativamente migliore rispetto all'altra classe. Tendenzialmente grazie al gioco SimUlme gli atteggiamenti ecologici relativi all'alimentazione sono migliorati. Il sapere ecologico è aumentato in entrambi i gruppi, tuttavia con accentuazioni diverse. I risultati depongono a favore dell'utilizzazione di adeguati giochi internet a scuola. 


\section{Internet-based environmental education: An experimental study applying the simulation game SimUlme in school}

\section{Summary}

An Internet based simulation game on ecological and economical consequences of nutrition consumption has been developed to improve environmental knowledge and attitudes in a playful and vivid way. In the experiment 12 classes were instructed on the consequences of nutrition consumption, 6 classes with application of the "SimUlme» learning game (experimental condition), 6 classes without it (control condition). The comprehensiveness, active participation, and engagement of the students in the experimental condition were judged significantly more positive than in the control condition. The tendency toward positive changes of environmental attitudes concerning nutrition behavior was more marked in the experimental condition as compared to the control condition. The lessons in the two conditions improved environmental knowledge in a complementary way. The results indicate that adequate Internet games can effectively support education in school. 